\title{
La Anestesiología Latinoamericana y su importancia como factor de desarrollo social
}

\author{
IGNACIO VARELA MALDONADO'
}

Con gran cariño y afecto para la Sociedad Chilena de Anestesiología y en especial a su nueva Editora en Jefe, gran amiga y colaboradora, Dra. María Carolina Cabrera Schulmeyer.

El desarrollo de la Anestesiología a partir de su descubrimiento por William Morton el 16 de octubre de 1846 es impresionante y factor primordial del desarrollo del acto quirúrgico y que ha beneficiado al propio desarrollo y perspectivas de vida a la humanidad.

Indudablemente la Anestesiología, su nacimiento, y su desarrollo han permitido que el hombre tenga lo que por siempre busco. El dolor siempre ha sido lo más temido y lo que más se ha tratado de evitar, es por ello que al tener la posibilidad de evitarlo fue y es visto actualmente como principal factor de desarrollo de la medicina.

Así mismo ha sido vital su participación en el desarrollo social de la población al permitir al paciente ser sometido a actos quirúrgicos cada vez más complejos y de mayor riesgo.

Actualmente no se concibe un acto anestésico sin la participación de un médico preparado y educado en la gran responsabilidad que conlleva el ejercicio de la Anestesiología.

Más el llegar a esta etapa no ha sido nada fácil pues aunque el dolor ha estado desde el origen de la vida ligado al hombre como ser vivo, son apenas ciento setenta y dos años en los que la anestesiología como especialidad médica ha tenido y se le ha reconocido en forma creciente su importancia y su necesidad.

Su inicio y desarrollo inicial fue más en forma romántica que profesional, sobre todo en nuestros países, en la que la responsabilidad del acto anestésico fue relegada inclusive a personas no profesionales de la medicina ni mucho menos.

Conforme el paso del tiempo la Anestesiología fue cada vez más requerida y reconocida como especialidad médica e igualmente se valoró más la importancia y la debida preparación necesaria del personal

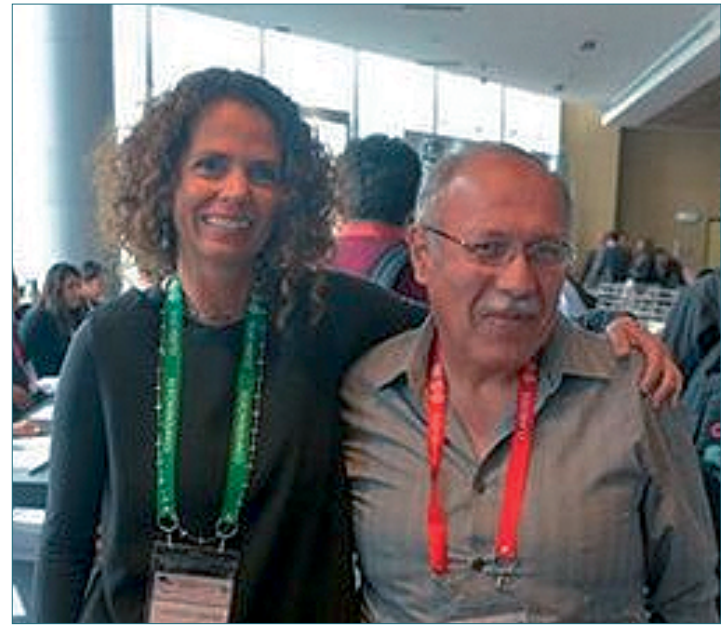

Figura 1.

médico que la aplicaba.

La anestesiología fue la primera especialidad médica en defender la seguridad del paciente como un enfoque específico. La coincidencia de múltiples factores que comenzó a fines de la década de 1970 provocó cambios significativos en la práctica que disminuyeron la mortalidad y la morbilidad catastrófica causada por la administración de anestesia[1].

A principios de la década de 1980, se hicieron disponibles avances importantes en la tecnología. El monitoreo electrónico que extendió los sentidos humanos (medición de oxígeno inspirado, capnografía y oximetría de pulso) permitió una monitorización continua genuina en tiempo real del suministro de oxígeno y la ventilación y oxigenación del paciente[1].

Situación paradójica en la historia de la Anestesiología cuando se nos narra por el génesis que Dios viendo que a Adán le faltaba una compañera lo durmió para sacarle una costilla y formar a Eva, de esa forma se realizó el primer acto anestésico. Y posteriormente tuvieron que pasar muchos años para que la humanidad lograra tener el conocimiento científico

Presidente de la Confederación Latinoamericana de Sociedades de Anestesia (CLASA) 2018 - 2019. 
de la Anestesiología.

El hombre inquieto por naturaleza necesitaba de un primer impulso para proseguir en su deseo de encontrar el alivio al dolor que le permitiera a su prójimo ser intervenido quirúrgicamente sin dolor y por ello el desarrollo científico de la Anestesiología se desarrolló en forma ascendente y cada vez con mayor seguridad para el paciente.

El 16 de octubre de 1846 William T. G. Morton utiliza el dietiléter en una demostración pública para producir anestesia quirúrgica.

En América Latina la inquietud y el deseo de quitar el dolor en el acto quirúrgico era igualmente buscado y de esta forma en 1847 el 29 marzo. Se administró la primera anestesia en un conflicto bélico durante la guerra México-americana en Veracruz, México, por el cirujano militar Edward H. Barton.

1847. Primera anestesia en Maracaibo, Venezuela por el Dr. Blas Villanueva por medio de la administración de éter a un hombre.

1847. El Dr. Roberto Haddock Lobo administra la primera anestesia en Río de Janeiro, Brasil. El paciente fue Francisco de Asís Páez Leme, se le administró éter.

1847. Primera anestesia con éter en Montevideo, Uruguay, el 2 de mayo, a un artillero herido a quien se le amputó un brazo en 4 minutos por el Dr. Patricio R.

1847. El Dr. Tulsbury administra la primera anestesia en Buenos Aires, Argentina. Eter para una corrección de estrabismo a un hombre de 30 años el 30 de agosto.

1847. El Dr. Vicente Antonio de Castro, administra la primera anestesia en La Habana, Cuba el 10 de marzo, administrando éter.

1847. El Dr. José Luna, el 30 de noviembre, administra la primera anestesia con éter en Guatemala, Guatemala, al paciente Urbano Paniagua.

1848. En Valparaíso, Chile, el 23 de octubre, el Dr. Francisco Javier Villanueva administra la primera anestesia en este país. Se realizó una amputación de miembro superior[2].

Dr. José Matilde Sansores practicó la eterización en un paciente en el Hospital General de San Juan de Dios de la ciudad de Mérida, Yucatán México, el día 4 de junio de 1847, tan sólo ocho meses después del experimento inicial de Morton en Boston[3].

La vida ha continuado su marcha, inexorable. Los procedimientos y los fármacos se han multiplicado y perfeccionado para hacer de la práctica anestesiológica una ciencia evolutiva y cada vez más segura aunque siempre perfectible[3].

A pesar de la distancia y de la poca oportunidad de tener información científica actualizada la Anestesiología a nivel mundial se fue desarrollando y al cabo de los siguientes 100 años su desarrollo era muy notorio y con bases científicas muy desarrolladas que permitieron a su vez que la cirugía igualmente se desarrollara al unísono.

Se puede afirmar con plena seguridad que conforme ha pasado el tiempo y las condiciones de trabajo, de conocimiento y de responsabilidad que la anestesiología ha escalado en forma cada vez más vertical un mayor reconocimiento a ella y a los profesionales del acto anestésico y se reconoce hoy que debe y es el líder en el manejo y en la seguridad del paciente.

Podemos estar seguros que es la anestesiología la especialidad médica donde con mayor vehemencia se manifiesta en el actuar del Anestesiólogo el que como menciona el Maestro Ignacio Chávez.

"El médico debe ser un hombre que se asoma sobre otro hombre, en un afán de ayudar, ofreciendo lo que tiene: un poco de ciencia y un mucho de comprensión y empatía".

Ser médico, que es -y nuestro deber radica, justamente, en que no deje jamás de serlo- la más noble y generosa vocación que el hombre ha inventado, a su paso por la Tierra, para asistir en su padecer a otro hombre[4].

"Debo recordar que vengo a expresarme como representante de una agrupación que lucha, que estudia. Que es integrada por mujeres y hombres probos, con la característica de ser un hombre que se asoma a otro hombre, que ayuda, que cuida, que ama"[5].

La evolución de la Anestesiología como especialidad médica en Latinoamérica ha sido en sus primeros cien años al cobijo y a la sombra de los conocimientos desarrollados en países con mayor índice de desarrollo científico por no haber investigación ni disponibilidad abundante de conocimientos de la especialidad.

A fines de los treinta inicio en la mayor parte de los países de la región un gran interés y preocupación por el estudiar y ser de parte de los médicos dedicados al ejercicio de esta especialidad en la educación médica continua que propicio la formación de organizaciones gremiales en forma de sociedades, asociaciones y colegios, la creación de la residencias médicas, publicaciones de la especialidad, cursos y congresos de Anestesiología y en forma más lenta por sus grandes costos en tener acceso a la tecnología en aparatos electro médicos que dieran un mayor índice de seguridad para los pacientes.

La formación de Residencias médicas y la educación médica continua se han constituido en la mejor forma de encontrar la superación y la difusión del conocimiento de la especialidad y de esta forma en los últimos años se ha incrementado notablemente la preocupación y el interés por mantener a los aneste- 
siólogos con un nivel de actualización de los conocimientos u con ello dar un mayor nivel de seguridad y beneficio a quien tiene que ser sometido por alguna razón a la aplicación de un acto anestésico.

\section{El Maestro Dr. Ignacio Chávez Expreso:}

Un deber fundamental de todo médico es estudiar y saber; saber adquirir conocimiento, para actuar el día de mañana en beneficio de los enfermos, y esto se puede resumir señalando la imperiosa necesidad de que el médico sea un científico actualizado, es decir, en estado constante de renovación y constatación de la vigencia de sus conocimientos. Esto constituye "el culto del saber", motor intemporal que subyace a la existencia como tal de un médico que se precie de serlo.

Si queremos saber, no debemos comenzar por ignorar".

El médico debe estudiar, si la curiosidad cientifica no basta para dictarle esa conducta, la ética profesional le exige ofrecer al enfermo una ayuda eficaz, no simulada. Quien por no estar enterado de los avances deja sin protección a su enfermo, comete en él un fraude que la ética profesional condena[6].

Por ello decimos que no hay mayor forma democrática y limpia de progreso de un pueblo que la enseñanza que le permitirá aplicar sus conocimientos en beneficio de todos.

En los últimos años la Anestesiología ha sido considerada como fundamental e indispensable para poder desarrollar los programas de salud pública y en el 2015 la Organización Mundial de Salud declaro como problema de salud mundial la falta de atención médico quirúrgico.

La cirugía viene siendo un componente esencial de la asistencia sanitaria en todo el mundo desde hace más de un siglo. Dada la creciente incidencia de los traumatismos, de cáncer y enfermedades cardiovasculares, el peso de la cirugía en los sistemas de salud públicos irá en aumento. Se calcula que en todo el mundo se realizan cada año 234 millones de operaciones de cirugía mayor, lo que equivale a una operación por cada 25 personas. Sin embargo, los servicios quirúrgicos están repartidos de manera desigual, pues el $75 \%$ de las operaciones de cirugía mayor se concentran en un $30 \%$ de la población mundial[7].

En enero de 2014, el presidente del Banco Mundial, Dr. Jim Yong Kim realizó un llamado a "compartir la visión y estrategia para la equidad global en la atención quirúrgica esencial", afirmando que "la cirugía es una parte indivisible e indispensable de la atención en salud".
Cada año se realizan en todo el mundo más de 234 millones de intervenciones quirúrgicas para una amplia gama de afecciones en pacientes de todas las edades y en todos los Estados Miembros. Las muchas afecciones que requieren atención quirúrgica son frecuentes y afectan a todos los grupos socioeconómicos y étnicos.

Las enfermedades que pueden tratarse quirúrgicamente se encuentran entre las 15 causas principales de discapacidad en todo el mundo. Según estimaciones conservadoras, un $11 \%$ de la carga de morbilidad mundial se debe a afecciones que pueden tratarse eficazmente mediante cirugía, y la proporción es aún mayor en los países de ingresos bajos y medianos[8].

Más de 100 millones de personas de todo el mundo sufren traumatismos y más de cinco millones de personas mueren por causa de la violencia y los traumatismos, y que el $90 \%$ de la carga mundial de mortalidad debida a la violencia y los traumatismos se registra en países de ingresos bajos y medianos; más de 289.000 mujeres mueren cada año al dar a luz y que alrededor de una cuarta parte de las muertes maternas y de las muertes y discapacidades de lactantes provocadas por la obstrucción del parto, hemorragias o infecciones podrían evitarse si se dispusiera universalmente de servicios quirúrgicos y anestésicos segu$\operatorname{ros}[9]$.

La causa de muerte a nivel mundial en el 2010 por enfermedades como el Paludismo, la tuberculosis o Síndrome de Inmuno deficiencia adquirida fueron 3.830.000 y en cambio por falta de atención quirúrgica anestésica oportuna se registran 18,6 millones de muertes. Existe un gran déficit de cirujanos, obstetras y de traumatólogos así como de anestesiólogos[10].

El informe Global Surgery 2030 (Cirugía Global 2030), publicado en la revista Lancet de agosto de 2015, sirvió como punto de referencia para describir el papel fundamental de la atención quirúrgica y anestésica en la mejoría de la salud de las personas y en la productividad económica personal y de los países[11].

El informe fue desarrollado por un equipo multidisciplinario de 25 comisionados y muchos otros colaboradores de más de 110 naciones, y contiene resultados sobre el estado de la atención quirúrgica en países de bajos y medianos ingresos (PBMI). Adicionalmente, contiene recomendaciones, indicadores y metas para perfilar la visión de cuál sería la mejor ruta para el acceso universal a la cirugía y la anestesia segura, económica y oportuna.

La atención de cirugía y anestesia se ha descuidado en muchos países de medianos y bajos ingresos. El tratamiento para las condiciones quirúrgicas, que 
representa una amplia gama de enfermedades que equivale aproximadamente el $30 \%$ de la carga mundial de enfermedad y abarca el $100 \%$ de las enfermedades sub-categóricas, queda fuera del alcance de la mayoría de la población mundial. Esto se traduce en la pérdida de la vida y la reducción del bienestar de millones de personas y frena el desarrollo económico de los países[11].

Estos estudios sirven para que:

- Los gobiernos en países de medianos y bajos ingresos puedan fortalecer los servicios quirúrgicos y sus sistemas nacionales de salud.

- Las organizaciones de desarrollo y de salud global puedan incluir indicadores de atención quirúrgica dentro de las metas de salud existentes y en los sistemas de vigilancia.

- Que organismos de financiamiento puedan invertir en la atención quirúrgica como vía para alivianar la pobreza y mejorar el bienestar común.

- Que socios internacionales puedan apoyar a los líderes locales en sus esfuerzos para proporcionar atención quirúrgica equitativa.

- Que el público en general conozca, difunda y promueva acciones para mayor acceso a servicios quirúrgicos para todos.

- En Latinoamérica la situación de falta de recursos anestésico quirúrgicos está alejada de los estándares marcados a menos de la mitad de los marcados por la OMS y las metas por alcanzar para el año 2030 son las siguientes:
La anestesiología en Latinoamérica se encuentra actualmente respondiendo con gran fuerza al reto de responder a la demanda de mayor número de profesionales de esta bella especialidad, con mayor unidad gremial y con una cada vez más mejor preparación, con adecuados programas de educación médica continua mediante Residencia inicialmente en Anestesia General y con programas de sub - especialidad, cursos, congresos, diplomados en los que se mantiene y se obtiene la gran responsabilidad de ser el médico responsable del manejo Preoperatorio, Transoperatorio y Postoperatorio lo que ha ocasionado que actualmente sea considerada con mayor especificidad como Medicina Perioperatoria.

Y aunque todavía el camino es largo y hay mucho por hacer podemos decir que en nuestros países cada vez más la Anestesiología está respondiendo a la gran demanda que se tiene como factor de desarrollo y de mejor estado de salud de la población de sus países. La Federación Mundial de Sociedades de Anestesia (WFSA) es quien a nivel mundial une gremialmente a las Sociedades de todo el mundo que trabajan en pro de la anestesiología y nos representa tanto a las Sociedades como a la Anestesiología como especialidad ante la Organización Mundial de Salud (OMS).

La Confederación Latinoamericana de Sociedades de Anestesia (CLASA) es quien representa regionalmente a la Anestesiología de 21 países miembro.

Ambas organizaciones unidas luchan a diario por ser quienes en un frente común trabajan con el mayor

\section{Indicadores principales para el monitoreo del acceso universal a servicios quirúrgicos y de anestesia que sean seguros, económicamente accesibles y oportunos \\ INDICADOR \\ OBJETIVO}

Acceso a cirugía esencial a tiempo

Densidad de fuerza laboral especializada en cirugía

Índice de mortalidad perioperatoria

Protección contra gastos empobrecedores

Protección contra gastos catastróficos
Lograr un mínimo de $80 \%$ en la cobertura de cirugía esencial y servicio de anestesia en cada país para el año 2030

Lograr que $100 \%$ de los países tengan 20 profesionales quirúrgicos. (Cirujanos, anestesiólogos y Gineco obstetras) por cada 100000 habitantes para el año 2030

Lograr que el $80 \%$ de los países vigilen el volumen quirúrgico para el 2020 y el $100 \%$ para el 2030 . Lograr un mínimo de 5000 procedimientos por 100000 habitantes para el año 2030

Lograr que el $80 \%$ de los países vigilen el índice de mortalidad perioperatoria para el 2020 y el $100 \%$ para el 2030 . En cada país, evaluar datos globales para el 2020 y establecer objetivos para el 2030

Lograr $100 \%$ de protección contra gastos empobrecedores pagados por fondos propios del paciente para atención quirúrgica o de anestesia para el año 2030

Lograr $100 \%$ de protección contra gastos catastróficos pagados por fondos propios del paciente para atención quirúrgica o de anestesia para el año 2030 
ahínco por el desarrollo, unidad y progreso científico y gremial de la especialidad. Y aprovechando las nuevas formas de comunicación nos mantiene unidos en torno a los mismos sueños e ideales.

La WFSA como interlocutora de la Anestesiología ha informado en torno a la declaración de la $68^{\text {ava }}$ Asamblea de la OMS celebrada en mayo de 2015 en que declaró como problema de salud mundial la falta de atención quirúrgica y de anestesia ha emitido el siguiente comunicado:

\section{Declaración global de la WFSA sobre los recursos humanos en Anestesiología}

Reivindicando la especialidad médica y reconociendo situaciones especiales pero bajo nuestro control.

- La Anestesia es compleja y potencialmente peligrosa.

- El cuidado óptimo del paciente depende de que la anestesia le sea administrada, dirigida o supervisada por un Anestesiólogo".

- Se aceptan situaciones especiales: "En otros países, especialmente aquellos con recursos limitados, las necesidades pueden lograrse, en parte, entrenando no-anestesiólogos".

- Las normas WFSA de seguridad, siempre están en proceso de actualización y reconocimiento por la OMS.

- El dilema acceso vs seguridad: lo que no debe plantearse.

- Promoción y defensa de la especialidad en varios frentes.

- Foros internacionales donde se difunda ampliamente la importancia de la especialidad y su gran responsabilidad, así como la debida preparación ante potenciales donantes, organizaciones quirúrgicas, gobiernos, pacientes y sus familias.

- De los gobiernos depende el tomar decisiones como los Planes Quirúrgicos Nacionales

Hay nuevos retos que afrontar y responder actualmente, la medicina institucional por falta de recursos humanos y económicos principalmente ha disminuido gradualmente su calidad y sobre todo su humanismo. No es lo mismo la calidad de atención en las grandes ciudades que en poblaciones pequeñas alejadas de centros hospitalarios de calidad. $Y$ actualmente también y por desgracia no es lo mismo la calidad de atención proporcionada por la medicina privada que la institucional.

Quien carece de recursos económicos se enfrenta en ocasiones a la disyuntiva de no encontrar en la medicina institucional la calidad de atención requerida y si acude a la medicina privada se encuentra en muchas de las veces comprometido a quedar con grandes deudas.

A pesar del paso del tiempo y que se reconoce cada vez más la importancia y la valía de la anestesiología, no ha logrado que sea reconocida por ello, ni su valía y aún entre los propios médicos impera mucho desconocimiento de ella.

Es importante que nosotros mismos logremos mayor conocimiento y respeto para la Anestesiología y lo obtendremos con nuestro profesionalismo, ética en el ejercicio diario, con el mantenimiento de la educación médica continúa, con cuidado y respeto a los pacientes y con el propio orgullo al ejercerla que lo lograremos

Entre la población en general permea mucho el desconocimiento sobre la especialidad ignorando que somos médicos, que hacemos estudios de residencia de varios años. En muchas ocasiones se duda y se pregunta si somos médicos y por ello siempre debemos presentarnos ante el paciente y sus familiares y llevar un correcto interrogatorio y exploración física y revisión del paciente, tener el consentimiento informado y disipar todas las dudas existentes.

\section{"UNA CONFIANZA FRENTE A UNA CONCIENCIA LOUIS PORTES"}

Los gobiernos de nuestros países si bien reconocen la falta de una atención médica oportuna y eficaz optan en muchas ocasiones por la preparación rápida y por lo mismo inadecuada de personal médico lo cual en lugar de dar una atención adecuada ocasiona que las complicaciones sean mucho mayores con un costo económico y humano mucho mayor.

Debemos participar en los programas de atención para la población, demostrar con hechos que la atención de calidad, con prontitud y eficacia es la mejor forma de elevar la productividad y calidad de vida.

Debemos demostrar que entre mayor sea la calidad de atención de vida de la población es menor el costo económico para el país, con gran satisfacción y ahorro económico para el país y para la propia población que ve elevado su nivel de vida.

Debemos los propios profesionales de la Anestesiología dar muestras de nuestra capacidad, dar atención de calidad y con muestras de respeto y amor por la especialidad y por nuestros pacientes.

Debemos sentirnos muy orgullosos de nuestra especialidad y de nuestras propias organizaciones gremiales a las cuales debemos honrar y alentar en su no- 
ble misión. Hagamos y pugnemos por su crecimiento y demostremos nuestro agradecimiento, lealtad y cariño a las propias instituciones que nos educaron y nos dieron los valores para el desempeño honroso de servir a nuestro prójimo en los momentos de mayor duda e incertidumbre del paciente temeroso e inquieto por su enfermedad que amerita ser sometido al acto anestésico previo al acto quirúrgico pero haciéndole ver que le será practicado por el médico que se ha preparado y que cada día desempeña su labor con gran dedicación y cariño.

Seamos los principales promotores de la grandeza de la más bella de las especialidades médicas.

Y siempre convencidos de lo afortunado que so- mos al ejercer la más noble y la más grande especialidad médica al ser responsables del cuidado de nuestro prójimo mediante el acto anestésico mientras es sometido a un acto quirúrgico.

Y como nos lo menciono el maestro Dr. Francisco Romo Salas Ex Presidente de la Federación Mexicana de Colegios de Anestesiología en la clausura del XLVIII Congreso Mexicano de Anestesiología Querétaro México septiembre de 2014.

\section{"ES UN PRIVILEGIO SER ANESTESIOLOGO"}

Fresnillo Zacatecas, México 28 de febrero de 2018

\section{Referencias}

1. Phisycian anesthesiologists Jan 28 - feb 3, 2018. Did you Know American Society Anesthesiology.

2. http://www.anestesia.com.mx/ histor2.html Luis Federico Higgins Guerra. Cronohistoriografía de la Anestesia.

3. Saenz Larrache Carlos. Rev Biomed Vol. 7/No. 3/Julio-Septiembre, 1996.

4. Guarner Vicente. Conferencia Dr. Ignacio Chávez. Acerca de la desaparición del arte de curar. Gac Méd Méx Vol. 138 No. 4, 2002.

5. Varela Maldonado Ignacio. Pa- labras en la Inauguración del L Curso anual de Anestesiología y Medicina Perioperatoria Colegio Mexicano de Anestesiología 3 de Julio 2014.

6. Chávez Ignacio. Deberes y responsabilidades del Médico de hoy frente a la Sociedad del futuro. Humanismo Médico, Educación y Cultura. Editorial de El Colegio Nacional. México 1978 pág 71 - 83.

7. La cirugía segura salva vidas - WHO/IER/PSP/2008.07 (c) Organización Mundial de la Salud, 2008.

8. $68^{\mathrm{a}}$ ASAMBLEA MUNDIAL DE LA SALUD A68/31 Punto 17.1 del orden del día provisional 20 de marzo de 2015.

9. $68^{\mathrm{a}}$ Asamblea mundial de la salud Ginebra, 18-26 de mayo de 2015 resoluciones y decisiones.

10. Vasco Ramírez Mauricio. Implicaciones del informe Global Surgery 2030 para el anestesiólogo Chairman del Comité de Anestesia Obstétrica y miembro del Council de la Federación Mundial de Sociedades de Anestesiología (WFSA) InfoSCARE 2017- IV.indd 13 Noviembre 2017-Enero 2018.

11. Global Surgery 2030: evidence and solutions for achieving health, welfare, and economic development. Lancet. 2015 Aug 8;386(9993):569-624. 